Introduction Spontaneous pneumothorax (SP) is common, with an estimated incidence of 5,000 per year in the UK. Primary spontaneous pneumothorax (PSP) can be managed with needle aspiration initially but those who fail to re-expand, and the majority of secondary SP (SSP), require admission to hospital, insertion of a chest drain and connexion to a bulky underwater drainage system. Patients with SSP have greater co-morbidities; have a long hospital stay (14-16 days) and a mortality of $\sim 16 \%$. There are no good predictors of outcome for patients with pneumothorax: those who will respond to drainage, those who have persistent air leak and those who will require surgery. Significant early air leak could be indicative of those patients who will not resolve spontaneously and will require surgery.

Aim To determine whether air leak measurement can predict patient outcome (surgical referral rate).

Methods Between December 2012 and June 2013, patients with pneumothorax managed on the Respiratory Ward of a tertiary referral centre had their "air leak" measured using a digital suction device (Thopaz, Medela UK).

Results A total of nine patients (6 SSP, 2 PSP, and 1 iatrogenic) were investigated. Air leak was measured during their in-patient stay: median of 3.5 days after admission (range 1-16 days). Three (33\%) were referred for surgical intervention for continued air leak and pneumothorax: their average air leak was $504 \mathrm{ml} / \mathrm{min}$ (range $222-952 \mathrm{ml} / \mathrm{min}$ ) compared to $77 \mathrm{ml} / \mathrm{min}$ (range $1-225 \mathrm{ml} / \mathrm{min}$ ) for the six patients not referred. The $427 \mathrm{ml} / \mathrm{min}$ difference was not statistically significant $(\mathrm{p}=0.2)$ with this sample size.

Conclusion At present, there are no good predictors of outcome for patients with pneumothorax. Air leak measurement postdrain insertion may be useful surrogate marker for on-going leak and hence non-resolving pneumothorax. This pilot study showed a difference in early leak measurement between those patients who were ultimately referred for surgery and those spontaneously resolving, and further larger studies are now warranted, comparing air leak to clinically important outcomes in pneumothorax.

\section{P219 EVALUATING MEDICAL THORACOSCOPY SERVICES AT A DISTRICT GENERAL HOSPITAL OVER THE PAST 13 YEARS}

${ }^{1} \mathrm{LM}$ George, ${ }^{2} \mathrm{HJ}$ Roberts, 'NJ Downer, 'GM Cox, ${ }^{1} \mathrm{NJ}$ Ali, ${ }^{1} \mathrm{ME}$ Roberts; 'Kingsmill Hospital, Sherwood Forest Hospitals NHS Trust, Mansfield, United KIngdom; ${ }^{2}$ Nottingham University Hospitals NHS Trust, Nottingham, United Kingdom

\subsection{6/thoraxjn-2013-204457.371}

Introduction Medical thoracoscopy is an invaluable tool in the management of undiagnosed and known malignant pleural effusions. In the UK, increasing numbers of centres are offering the procedure We present the experience from a single district general hospital that has been offering medical thoracoscopy over the last 13 years.

Methodology A retrospective analysis of 411 case notes of patients who had a medical thoracoscopy between July 2000 and April 2013.

Results 291 patients (71\%) were male. The median age was 70 years (24 to 92). In 321 patients (78\%) the procedure was done for diagnostic purposes and the procedure was done on the right side $(20 \%)$ had lung cancer, 94 (23\%) had mesothelioma and 66 ( 16\%) had other cancer.

In the 231 patients that had operator observations as appearing malignant $198(86 \%)$ were confirmed on histology. 34 ( $30 \%$ ) patients had malignancy in operator observations that

\section{Diagnostic rates}

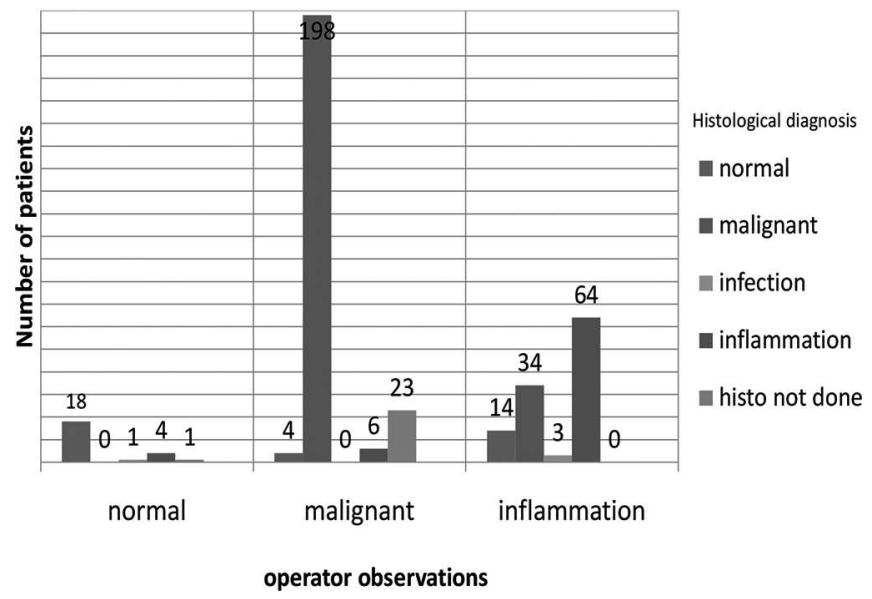

Abstract P219 Figure 1.

were reported as inflammation but reassuringly no patients had malignancy in the operator observations that were reported as appearing normal. Talc poudrage was done in 261 ( 64\%) patients. 53 patients (13\%) required a further pleural intervention within 12 months. Complications included empyema in 3 $(0.7 \%)$, renal failure in $6(1.4 \%)$ and trapped lung in $20(5 \%)$ patients. 6 patients $(1.4 \%)$ died within 15 days of the procedure and major contributors to mortality were hospital acquired infection.

Conclusions Medical thoracoscopy is an effective procedure for diagnosing and managing pleural effusions. It is associated with a low complication rate and can be performed in patients with poor performance status. Caution should be exercised in patients with multiple comorbidities and careful management of cardiovascular status post-operatively could help reduce post-procedure kidney injury.

\section{P220 EVOLVING ROLE OF THE RESPIRATORY SPECIALIST: PLEURAL ULTRASOUND SERVICE 4 YEARS ON}

B Khan, H Aminy Raouf, M Mushtaq; Darent Valley Hospital, Dartford, UK

\subsection{6/thoraxjnl-2013-204457.372}

Background The timely and safe investigation, intervention and management of pleural effusions remains discrepant with varying practices. Following the National Patient Safety Agency 2008 report highlighting 12 deaths from intercostal chest drain, the BTS recommended using ultrasound guidance when inserting chest drains. However, conventionally diagnostic and therapeutic thoracentesis have routinely been performed with either no image guidance or an "X mark the spot" in the radiology department and then transferred back to the ward for the actual procedure. All of these give rise to concern and possibly impact upon quality of care and patient safety.

Methods A retrospective analysis of $\sim 4$ years experience of providing a Respiratory team delivered ultrasound pleural service; both "inpatient" and "ambulatory".

Results In the 12 months prior to the establishment the pleural service, the radiology department did a total of 96 ultrasounds, of which 46 were " $X$ " marks the spot.

Since May 2010, 581 pleural ultrasounds have been performed; with only 5 dry taps and 3 clinically insignificant iatrogenic pneumothoraces. $41 \%$ were therapeutic thoracentesis, 
$26 \%$ diagnostic, $18 \%$ ultrasound only with no intervention, and $14 \%$ pre chest drain insertion or thoracoscopy. " $\mathrm{X}$ " marks the spot are now obsolete.

Conclusions Pleural ultrasound has gradually but surely become an essential component of the Respiratory specialist's remit. An inpatient pleural service enables prompt assessment and diagnosis, relief of symptoms and onward management as appropriate. With an ambulatory pleural service, patients with either known malignant pleural effusion or first presentation can be managed as elective daycase procedures without attending $A \& E$ or being admitted. Once symptoms are relieved patients are able to go home knowing how to access the Service if the fluid recurs without a crisis admission via A\&E. Moreover, unnecessary invasive pleural interventions can be avoided and definitive management expedited.

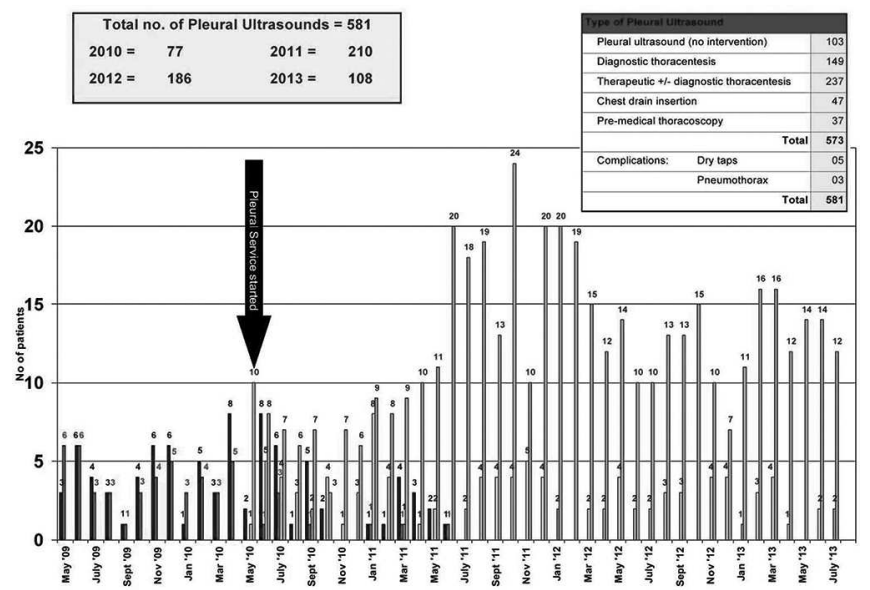

Abstract P220 Figure 1.

Though requiring appropriate work planning and resources it does result in substantial qualitative and quantitative improvements in patient care. No doubt, not all pleural effusions need be aspirated under ultrasound guidance, however, this is not an exact science and not infrequently, with the aid of pleural ultrasound, an invasive pleural intervention may be avoided altogether.

\section{P221 IMPROVED MANAGEMENT OF PLEURAL EFFUSION IN A DISTRICT GENERAL HOSPITAL}

${ }^{1} \mathrm{P}$ Shetty, ${ }^{2} \mathrm{SM}$ Menzies, ${ }^{1} \mathrm{~J}$ Ojoo; ${ }^{1}$ Northampton General Hospital, Northampton, UK; ${ }^{2}$ Wexham Park Hospital, Slough, UK

\subsection{6/thoraxjnl-2013-204457.373}

Background Exudative pleural effusion (EPE) is a common presenting problem, associated with serious underlying pathology warranting expeditious and thorough investigation. The BTS guideline outlines a diagnostic algorithm and recommends referral of patients to chest physicians following inconclusive initial pleural fluid analysis.

We have carried out a series of audits on the management of EPE in a district general hospital (DGH). The first revealed deficiencies in the diagnostic pathway, with low diagnostic rates compared with published data. We instituted regular teaching sessions including simulated training of junior doctors, established a specialist pleural effusion clinic (SPEC) and during the same period there was increase in the number of respiratory registrars. In a previous study comparing results of the first retrospective audit cohort (RC) to the SPEC cohort showed improved outcomes in the latter. Unfortunately a significant proportion of patients with EPE are still diagnosed on acute admission. In this audit we compare the management of these patients to the RC.

Methods We carried out a retrospective re-audit (RA), against BTS guidelines, of non-elective admissions to the general medical take from January to December 2011 with EPE. Clinical records of patients with pleural effusion were reviewed and analysed for investigations, involvement of respiratory physician, length of stay and outcome. Those with transudate effusions were excluded. This was similar to the RC which covered the period from February 2005 to June 2006. We present comparative results.

Results Of 106 patients, 66 patients had diagnostic aspiration, 18 had small effusions unable to aspirate, 19 had known diagnoses and 3 had terminal cancer. The respective results in the RA compared to the RC showed that mean age was 65 vs 68 years, $86 \%$ had all the recommended tests vs $46 \%, 87 \%$ had chest physician input vs $50 \%$ and diagnosis confirmed in $95 \%$ vs $58 \%$. The median length of stay in hospital was 4 days (range $0-51$ ) vs 12 days (range 1-55). Table 1 demonstrates pleural fluid tests performed.

Conclusions The RA shows improved investigation, access to chest physician, diagnostic rates and average length of hospital stay in patients with EPE in this DGH. The change is likely multifactorial owing to increasing awareness, training, and better specialist services.

\begin{tabular}{lll}
\multicolumn{3}{l}{ Abstract P221 Table 1. Pleural fluid investigations } \\
\hline Test on pleural fluid & RC (\% of samples sent) & Re-audit (\% of sample sent) \\
Protein & $91 \%$ & $97 \%$ \\
LDH & $91 \%$ & $94 \%$ \\
pH & $55 \%$ & $94 \%$ \\
MCS/ AFB & $85 \%$ & $100 \%$ \\
Cytology & $82 \%$ & $95 \%$ \\
\hline
\end{tabular}

\section{P222 PLEURAL TB: A COMMON CAUSE OF PLEURAL EFFUSION IN SOUTH LONDON}

${ }^{1} \mathrm{O}$ Pickering, ${ }^{2} \mathrm{R}$ Sarefuji, ${ }^{2} \mathrm{~L}$ Ahmed, ${ }^{2} \mathrm{R}$ Breen, ${ }^{2} \mathrm{H}$ Milburn; ${ }^{1}$ King's College London, London, England; 'Guy's and St Thomas' NHS Foundation Trust, London, England

\subsection{6/thoraxjnl-2013-204457.374}

Background Pleural tuberculosis (TB) is one of the most common forms of extrapulmonary TB, reported to account for up to $25 \%$ of TB infected adults, and $30 \%$ of exudative pleural effusions in developing countries. Despite this, little information has been reported on its incidence within London.

Aims and methods A retrospective observational study was performed at a London tertiary referral centre. The aim was to identify the contribution of pleural TB to the overall burden of both pleural and TB disease, and assess diagnostic yields of investigation techniques and outcomes of treatment.

Results 28 patients were diagnosed with pleural TB between Jan $1^{\text {st }} 2010-31^{\text {st }}$ Dec 2012. This represented $6.2 \%$ of the total number of TB cases, and $4 \%$ of the total number of investigated pleural disease cases. The mean age of the patients was $33 \pm 10$ (range 17-62); 79\% were male; 46\% were Black African, 29\% Asian, 21\% Caucasian and 4\% Black Caribbean. There was a range of symptoms at presentation, with $96 \%$ of patients complaining of at least one symptom (Figure 1). 